\title{
○
}

\section{An unending ode to pneumonia}

I must first note that due homage should be paid to the Internet and

Google, clearly the editorialist's best friends.

In 1903, Dr. Arthur R. Reynolds, the Chicago Commissioner of Health, published an article in JAMA' ${ }^{1}$ entitled "Pneumonia: the new 'captain of the men of death.' Its increasing prevalence and the necessity of methods for its restriction." He wrote, "Figures are hard reading and harder hearing; but I must ask you to listen to a few in order that you may comprehend the magnitude of the pneumonia problem as I view it." He then noted that Professor William Osler, in the 1901 edition of his Principles and Practice of Medicine, ${ }^{2}$ said of pneumonia: "The most widespread and fatal of all acute infectious diseases, pneumonia, is now the "Captain of the Men of Death."

Osler, who it seems attributed most acute bacterial pneumonia to the Pneumococcus, borrowed and amended this phrase from the 17th century Baptist preacher and philosopher John Bunyan. ${ }^{3}$ That he knew of this verbiage, without access to the Internet, is testimony to Osler's keen literary as well as medical knowledge. Bunyan was referring to tuberculosis when he wrote in 1680 (in The Life and Death of Mr. Badman ${ }^{3}$ ), "He was dropsical, he was consumptive, he was surfeited, was gouty, and, as some say, he had a tang of the Pox in his Bowels. Yet the Captain of all these Men of Death that came against him to take him away, was the Consumption, for "twas that that brought him down to the Grave."

The face of pneumonia has changed slowly over the centuries, although far more rapidly over the past few decades. But as Modi and Kovacs note in this issue, ${ }^{4}$ community-acquired pneumonia remains a major health issue, and its management is still a component of bread-and-butter medicine, as tuberculosis (consumption) has faded in frequency but has clearly not disappeared.

As our therapeutic and diagnostic armamentaria have grown, so has our understanding of the principles of pathophysiology and management of bacterial pneumonia, but there are still clear limitations to our knowledge and practice skills. Only some of those limitations stem from recognition of apparently new respiratory pathogens and syndromes (eg, Legionella, AIDS, Hantavirus, SARS, novel coronavirus). Frontline providers still face many of the same challenges and questions that Bass editorialized on in Chest in $1985^{5}$ and that we struggled with as residents earlier that same decade: When should a patient with suspected pneumonia be admitted? How can we reliably recognize the patient with early pneumonia? How can we identify the likely bug and choose an appropriate antibiotic? And how long should antibiotics be administered? How should we factor into our decision the local antibiotic resistance pattern and the patient's comorbidities? (Regarding comorbidities, a forthcoming paper in the Journal will discuss the effect of heavy alcohol use on management of pneumonia.)

Reading through the different risk-stratification scores codified to help standardize clinical decision-making reminds me of emergency-room discussions between we residents (our fingers and white pants stained with Gram stain crystal violet) and our attending physician as to whether the patient was "sick enough" to need admission, or 
whether we should intravenously hydrate and then repeat the examination and chest $\mathrm{x}$-ray to see if rales or infiltrate would appear before making that decision. The American Thoracic Society and Infectious Diseases Society of America ${ }^{6}$ opine that use of clinical prediction rules enhances clinical judgment in making the appropriate decision to admit or provide outpatient therapy. I can see the rolling of eyes from several of my former ER attendings as to the need for this, but I can also recall scenarios where the availability of a validated tool to support the safety of outpatient treatment would have been most welcome.

At the end of the day, rules or not, in 2020 just as in 1980 (and 1903), the accurate examination, clinical history, and under certain circumstances the sputum Gram stain and culture results still factor into the gestalt of clinical judgment when dealing with the Captain.

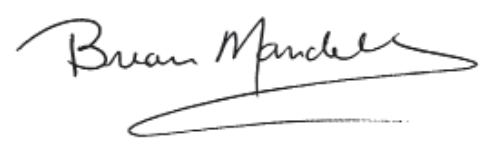

BRIAN F. MANDELL, MD, PhD Editor in Chief

1. Reynolds AR. Pneumonia: the new "captain of the men of death." Its increasing prevalence and the necessity of methods for its restriction. JAMA 1903; XL(9):583-586. doi:10.1001/jama.1903.92490090031001

2. Osler W. Principles and Practice of Medicine, Designed for the Use of Practitioners and Students of Medicine. 4th Ed. New York, D. Appleton and Company, 1901.

3. Bunyan J. The Life and Death of Mr. Badman, Presented to the World in a Familiar Dialogue Between Mr. Wiseman, and Mr. Attentive. London, Printed by J.A. for Nath. Ponder at the Peacock in the Poultrey, neer the Church, 1680.

4. Modi AR, Kovacs CS. Community-acquired pneumonia: strategies for triage and treatment. Cleve Clin J Med 2020; 87(3):145-151. doi:10.3949/ccjm.87a.19067

5. Bass JB Jr. Lieutenant of the men of death. Chest 1985; 88:483-484. doi:10.1378/chest.88.4.483

6. Metlay JP, Waterer GW, Long AC, et al. Diagnosis and treatment of adults with community-acquired pneumonia. An official clinical practice guideline of the American Thoracic Society and Infectious Diseases Society of America. Am J Respir Crit Care Med 2019; 200(7):e45-e67. doi:10.1164/rccm.201908-1581ST 